\title{
Die neuen Volkskrankheiten
}

$\mathrm{P}$ sychische Störungen gehören zu den häufigsten Krankheiten. Allein in Deutschland leiden rund acht Millionen Menschen an einer behandlungsbedürftigen psychischen Störung. Nach einer Schätzung der Weltgesundheitsorganisation (WHO) werden Depressionen bis zum Jahr 2020 der führende Grund für Invalidität und vorzeitigen Tod sein. Dicht auf liegen Angsterkrankungen, die bereits heute eine Lebenszeitprävalenz von bis zu 25 \% aufweisen. Aufgrund der Bevölkerungsstruktur ist außerdem in den nächsten Jahrzehnten mit einer Verdoppelung bis Verdreifachung der Anzahl von Demenzerkrankungen zu rechnen. Diese Ausgabe der psychoneuro will einen prägnanten Überblick über diese neuen Volkskrankheiten und ihre Behandlung geben.

Insbesondere die Behandlung mit Psychopharmaka steht zurzeit in den Medien unter Dauerbeschuss. Antidepressiva sollen Suizide auslösen, Antidementiva seien unwirksam und teure Antipsychotika nicht besser als die alten. Kann man wirklich noch guten Gewissens verordnen oder ist alles nur Geldmacherei und Medienrummel?

Prof. Jürgen Fritze nimmt Stellung zu Antidepressiva und räumt mit den Vorurteilen auf. Derzeit gibt es keine Evidenz, dass Antidepressiva das Suizidrisiko erhöhen. Nicht auszuschließen sei, dass Antidepressiva zu Therapiebeginn das Risiko von suizidalen Gedanken und Suizidversuchen erhöhen, möglicherweise eher SSRI als andere. Deshalb muß den Patienten zu Therapiebeginn besondere Aufmerksamkeit gewidmet werden. Das gilt insbesondere für Kinder und Jugendliche, für die bisher ein eindeutiger Wirksamkeitsnachweis nicht erbracht wurde, wahrscheinlich wegen des hier besonders hohen Plazebo-Effektes. Insbesondere weil es sich um seltene Ereignisse zu Therapiebeginn handelt, können doppelblinde Langzeitstudien nichts zur weiteren Klärung beitragen, zumal sie ethisch unvertretbar wären. Vielmehr muss der Pharmakovigilanz erhöhte Aufmerksamkeit gewidmet werden.
Dietmar Winkler, Edda Pjrek, Siegfried Kasper, Wien, geben einen kurzen Überblick über uni- und bipolare Depressionen und versuchen die Frage, wann Psychotherapie, wann Pharmakotherapie, zu beantworten.

Dass es nicht nur schwarz und weiß gibt, zeigt auch Georg Adler, Mannheim, in seinem Beitrag zu Demenzerkrankungen. Bei der Alzheimer-Demenz spielen vaskuläre Faktoren eine wichtige Rolle. Die zerebralen Mikrogefäße scheinen an der Entstehung der Erkrankung beteiligt zu sein. Für die Praxis bedeutet dies, dass auch vaskuläre Risikofaktoren bei der Diagnostik überprüft und gegebenenfalls behandelt werden sollten.

Die z.Zt. absehbaren, wichtigsten Entwicklungen für die Zukunft anxiolytischer Pharmakotherapie stellen Dirk Wedekind und Borwin Bandelow, Göttingen, vor. Neuropeptid- und Neurosteroid-Rezeptor-Liganden, spezifische Modulatoren am GABA-A-BenzodiazepinRezeptorkomplex und Modulatoren des glutamatergen sowie des serotonergen Systems können vielleicht schon bald die Palette der Behandlungsmöglichkeiten ergänzen.

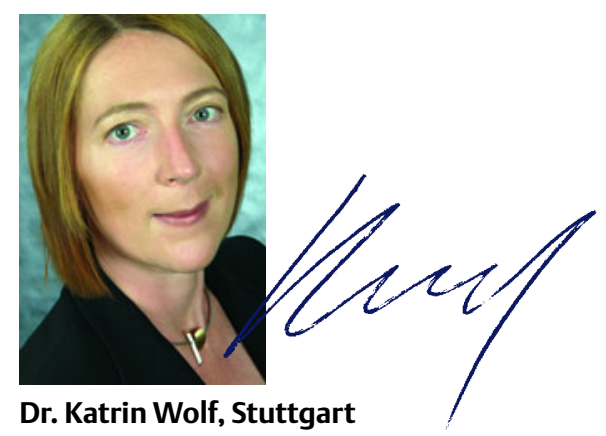

